Annals of Pure and Applied Mathematics

Vol. 17, No. 1, 2018, 67-83

ISSN: 2279-087X (P), 2279-0888(online)

Published on 25 April 2018

Annals of

www.researchmathsci.org

DOI: http://dx.doi.org/10.22457/apam.v17n1a8

Pure and Applied

Mathematics

\title{
On Generalized Bounded Mocanu Variation Related to $q$-Derivative and Conic Regions
}

\author{
Hamid Shamsan ${ }^{1}$ and S. Latha ${ }^{2}$
}

Department of Mathematics, Yuvaraja's College

University of Mysore, Mysore 570 005, INDIA

hmas19771@gmail.com and drlatha@gmail.com

Received 18 March 2018; accepted 22 April 2018

Abstract. In this paper, we introduce a class of normalized analytic functions defined in the open unit disc. We study the unified class of functions with bounded Mocanu variation which map the open unit disk onto conic regions using $q$-derivative. We invistigate several Interesting mapping properties, certain inclusion results and generalized type of $q$-Bernardi integral operator for this class.

Keywords: $q$-derivative, Conic regions, $q$-Bernardi integral, k-uniformly $q$-convex functions, k-uniformly $q$-starlike functions.

AMS Mathematics Subject Classification (2010): 30C45

1. Introduction

Let $A$ denote the class of functions of form

$$
f(z)=z+\sum_{n=2}^{\infty} a_{n} z^{n}
$$

which are analytic in the open unit disk $U=\{z: z \in \mathrm{C}$ and $|z|<1\}$,

and $S$ denote the subclass of $A$ consisting of all function which are univalent in $U$.

Noor and Malik [14] introduced the classes of generalized bounded Mocanu variation associated with conic domains which generalize many classes studied in literature by $\mathrm{R} \ddot{o}$ nning, Goodman, Kanas, Shams and Wisniowska.

The aim of our result is to generalize the class of functions with bounded Mocanu variation which map the open unit disk onto conic domains. Interesting properties of these functions are investigated.

Let $f, g \in \mathrm{A}$. If there exists a function $\phi(z)$ with $\phi(0)=0,|\phi(z)|<1, z \in \mathrm{U}$, such that $f(z)=g(\phi(z))$, then we say $f(z)$ is subordinate to $g(z)$ and we denote this by $f \prec g$. Jackson[8] initiated $q$-calculus and developed the concept of the $q$-integral and $q$-derivative.

For a function $f \in \mathrm{S}$ given by (1.1) and $0<q<1$, the $q$-derivative of $f$ is defined by 
Hamid Shamsan and S. Latha

$$
\partial_{q} f(z)=\left\{\begin{array}{cc}
\frac{f(z)-f(q z)}{z(1-q)}, & z \neq 0, \\
f^{\prime}(0), & z=0 .
\end{array}\right.
$$

Equivalently (1.2), may be written as

where

$$
\partial_{q} f(z)=1+\sum_{n=2}^{\infty}[n]_{q} a_{n} z^{n-1}, \quad z \neq 0
$$

$$
[n]_{q}=\frac{1-q^{n}}{1-q} .
$$

Note that as $q \rightarrow 1, \quad[n]_{q} \rightarrow n$. For a function $f(z)=z^{n}$, we can observe that

Then

$$
\partial_{q} f(z)=\partial_{q}\left(z^{n}\right)=\frac{1-q^{n}}{1-q} z^{n-1}=[n]_{q} z^{n-1} .
$$

$$
\lim _{q \rightarrow 1} \partial_{q} f(z)=\lim _{q \rightarrow 1}[n]_{q} z^{n-1}=n z^{n-1}=f^{\prime}(z),
$$

where $f^{\prime}(z)$ is the ordinary derivative. For more properties of see[3, 5]. As a right inverse, Jackson[9] presented the $q$-integral of a function $f$ as

$$
\int_{0}^{z} f(t) d_{q} t=z(1-q) \sum_{k=0}^{\infty} q^{k} f\left(z q^{k}\right)
$$

provided that the series converges. For a function $f(z)=z^{k}$, we note that

and

$$
\int_{0}^{z} f(t) d_{q} t=\int_{0}^{z} t^{k} d_{q} t=\frac{z^{k+1}}{[k+1]_{q}} \quad(k \neq-1)
$$

$$
\lim _{q \rightarrow 1} \int_{0}^{z} f(t) d_{q} t=\lim _{q \rightarrow 1} \frac{z^{k+1}}{[k+1]_{q}}=\frac{z^{k+1}}{k+1}=\int_{0}^{z} f(t) d t,
$$

where $\int_{0}^{z} f(t) d t$ is the ordinary integral.

Under the hypothesis of the definition of q $\hat{a}^{\wedge}$ 'difference operator, then we have the following rules.

(i) $\partial_{q}(a f(z) \pm b g(z))=a \partial_{q} f(z) \pm b \partial_{q} g(z)$, where $a$ and $b$ any real (or complex) constants

(ii) $\partial_{q}(f(z) g(z))=g(q z) \partial_{q} f(z)+f(z) \partial_{q} g(z)=f(z) \partial_{q} g(z)+\partial_{q} f(z) g(q z)$

(iii) $\partial_{q}\left(\frac{f(z)}{g(z)}\right)=\frac{g(z) \partial_{q} f(z)-f(z) \partial_{q} g(z)}{g(q z) g(z)}$

Denote by $P(q)(0<q<1)$ the family of functions of the form $p(z)=1+p_{1} z+p_{2} z^{2}+\ldots$ regular in the open unit disc $U$ and satisfying 
On Generalized Bounded Mocanu Variation Related to $q$-Derivative and Conic Regions

$$
\left|p(z)-\frac{1}{1-q}\right|<\frac{1}{1-q}, \quad z \in \mathrm{U}, \quad q \in(0,1) \text {. }
$$

Definition 1.1. A function $f(z) \in \mathrm{A}$ is said to be in the class $k-U B_{q}(\alpha, \beta, \gamma), k \geq 0$, if and only if, for $\alpha \geq 0,0 \leq \beta<1,0 \leq \gamma<1,0<q<1$,

$$
\operatorname{Re}\left\{J_{q}(\alpha, \beta, \gamma, f(z))\right\}>k\left|J_{q}(\alpha, \beta, \gamma, f(z))-1\right| .
$$

where

$$
J_{q}(\alpha, \beta, \gamma, f(z))=\frac{1-\alpha}{1-\beta}\left\{\frac{z \partial_{q} f(z)}{f(z)}-\beta\right\}+\frac{\alpha}{1-\gamma}\left\{1-\gamma+\frac{q z \partial_{q}^{2} f(z)}{\partial_{q} f(z)}\right\} .
$$

We note that:

(i) $\lim _{q \rightarrow 1^{-}} k-U B_{q}(\alpha, \beta, \gamma)=k-U B(\alpha, \beta, \gamma)$, (Noor and Malik [14]);

(ii) $\lim _{q \rightarrow 1^{-}} k-U B_{q}(0,0, \gamma)=k-S T$ and $\lim _{q \rightarrow 1^{-}} k-U B_{q}(1, \beta, 0)=k-U C V$, (Kanas and Wisniowska [11, 12]);

(iii) $\lim _{q \rightarrow 1^{-}} k-U B_{q}(0, \beta, \gamma)=S D(k, \beta)$ and

$\lim _{q \rightarrow 1^{-}} k-U B_{q}(1, \beta, \gamma)=K D(k, \gamma)$, (Shams et al. [15]);

(iv) $\lim _{q \rightarrow 1^{-}} k-U B_{q}(\alpha, 0,0)=U M(\alpha, k)$, (Kanas [15]);

(v) $\lim _{q \rightarrow 1^{-}} 0-U B_{q}(\alpha, 0,0)=M_{\alpha}$, (Mocanu [13]).

Geometrically, a function $f \in \mathrm{A}$ is said to be in the class $k-U B_{q}(\alpha, \beta, \gamma)$, if and only if, the function $J_{q}(\alpha, \beta, \gamma, f(z))$ takes all values in the conic domain $\Omega_{k}$ which is defined as

$$
\Omega_{k}=\left\{u+i v: u>k \sqrt{(u-1)^{2}+v^{2}}\right\}
$$

This domain represents the right half plane when $k=0$, a hyperbola when $0<k<1$, a parabola when $k=1$ and an ellipse when $k>1$. The functions, which play the role of extremal functions of these conic regions are given as follows

$$
p_{k}(z)=\left\{\begin{array}{c}
\frac{1+z}{1-z}, \quad k=0, \\
1+\frac{2}{\pi^{2}}\left(\log \frac{1+\sqrt{ } z}{1-\sqrt{ } z}\right)^{2}, k=1 \\
1+\frac{2}{1-k^{2}} \sinh ^{2}\left[\left(\frac{2}{\pi} \arccos k\right) \arctan h \sqrt{ } z\right], 0<k<1, \\
\frac{1}{k^{2}-1} \sin \left(\frac{\pi}{2 R(t)} \int_{0}^{\frac{u(z)}{\sqrt{t}}} \frac{1}{\sqrt{ } 1-x^{2} \sqrt{ } 1-(t x)^{2}} d x\right)+\frac{1}{k^{2}-1}, k>1,
\end{array}\right.
$$


Hamid Shamsan and S. Latha

where $u(z)=\frac{z-\sqrt{ } t}{1-\sqrt{ } t z}, t \in(0,1) z \in U$ and $z$ is chosen such that $k=\cosh \left(\frac{\pi R^{\prime}(t)}{4 R(t)}\right)$, $R(t)$ is the Legendre's complete elliptic integral of the first kind and $R^{\prime}(t)$ is complementary integral of $R(t),[11,12]$. Equivalently a function $f \in A$ is said to be in the class $k-U B_{q}(\alpha, \beta, \gamma), k \geq 0$, if and only if, $J_{q}(\alpha, \beta, \gamma, f(z)) \prec p_{k}(z)$, where $p_{k}(z)$ is defined by (1.5), $\alpha \geq 0,0 \leq \beta<1,0 \leq \gamma<1$.

By virtue of the properties of the domains for $p \prec p_{k}$, we have

$$
\mathfrak{R} p(z) \geq \mathfrak{R}\left(p_{k}(z)\right)>\frac{k}{k+1} .
$$

Lemma 1.2. (Jack's lemma)[7] Let $B(z)$ be analytic in $U$ with $B(0)=0$. If the maximum value of the $|B(z)|$ on the circle $|z|=r,(0<r<1)$ is attained at $z=z_{0}$, then there is a real number $\lambda, \lambda \geq 1$, such that

$$
\frac{z_{0} B^{\prime}\left(z_{0}\right)}{B\left(z_{0}\right)}=\lambda
$$

Lemma 1.3. If $f(z) \prec H(z)$ and $g(z) \prec H(z)$, then for $t \in[0,1]$,

$$
(1-t) f(z)+\operatorname{tg}(z) \prec H(z) \text {. }
$$

\section{Main results}

We need the following lemmas, to prove our main results.

Lemma 2.1. ( $q$-Jack's lemma)[17] Let $B(z)$ be analytic in $U$ with $B(0)=0$. If the maximum value of the $|B(z)|$ on the circle $|z|=r$, is attained at $z=z_{0} \in \bigcup$, then there is a real number $\lambda, \lambda \geq 1$, such that

$$
\frac{z_{0} \partial_{q} B\left(z_{0}\right)}{B\left(z_{0}\right)}=\lambda
$$

Proof: Using the definition of $q$-difference operator and Jackâ€ ${ }^{\mathrm{TM}_{\mathrm{S}}}$ Lemma (1.2), then we have

$$
\partial_{q} B(z)=\frac{B(z)-B(q z)}{(1-q) z}=\frac{B(z)-B\left(z_{0}\right)}{\left(z-z_{0}\right)}, \quad q z=z_{0} .
$$

If we take the limit for $z \rightarrow z_{0}$ we get

Therefore we have

$$
\lim _{z \rightarrow z_{0}} \partial_{q} B(z)=\partial_{q} B\left(z_{0}\right)=\lim _{z \rightarrow z_{0}} \frac{B(z)-B\left(z_{0}\right)}{\left(z-z_{0}\right)}=B^{\prime}\left(z_{0}\right) .
$$

$$
z_{0} B^{\prime}\left(z_{0}\right)=\lambda B\left(z_{0}\right)=z_{0} \partial_{q} B\left(z_{0}\right) \text {. Hence the result. }
$$

Lemma 2.2. [4] Let $f(z) \in \mathrm{A}$, then

$$
\partial_{q}\{\log (f(z))\}=\frac{\ln q}{(q-1)} \frac{\partial_{q} f(z)}{f(z)}
$$


On Generalized Bounded Mocanu Variation Related to $q$-Derivative and Conic Regions Proof: $\quad \partial_{q}\{\log (f(z))\}=\partial_{q} \log (f(z)) \partial_{q} f(z)$,

but

Therefore

$$
\partial_{q} \log (f(z))=\frac{\log (q f(z))-\log (f(z))}{(q-1) f(z)}=\frac{\ln q}{(q-1) f(z)} .
$$

$$
\partial_{q}\{\log (f(z))\}=\frac{\ln q}{(q-1)} \frac{\partial_{q} f(z)}{f(z)} .
$$

Lemma 2.3. Let $p(z)=1+p_{n} z^{n}+\ldots, \quad n \geq 1$, be analytic in the unit disc $\bigcup$ and let $g(z)=1+c_{1} z+\ldots$, be analytic and univalent in $\overline{\mathrm{U}}$. If $p(z)$ is not subordinate to $g(z)$, then there exists a real number $\lambda(\lambda \geq 1), z_{0} \in \mathrm{U}$ and $\zeta_{0} \in \partial U$ such that

1. $p\left(|z|<\left|z_{0}\right|\right) \subset g(\bigcup)$.

2. $p\left(z_{0}\right)=g\left(\zeta_{0}\right)$.

3. $\arg \left(z_{0} \partial_{q} p\left(z_{0}\right)\right)=\arg \left(\zeta_{0} \partial_{q} g\left(\zeta_{0}\right)\right)$.

4. $\left|z_{0} \partial_{q} p\left(z_{0}\right)\right|=\lambda\left|\zeta_{0} \partial_{q} g\left(\zeta_{0}\right)\right|$.

Proof: Suppose, $p(z)$ is not subordinate to $g(z)$, then there is $z_{0} \in \bigcup$ such that $p\left(|z|<\left|z_{0}\right|\right) \subset g(\mathrm{U})$ and $p\left(z_{0}\right) \in \partial g(\mathrm{U})$. Now let us define $F(z)=g^{-1}(p(z))$, then $F(z)$ satisfies the hypotheses of Lemma 2.1 at $z=z_{0}$, and hence the result.

Lemma 2.4. Let $\beta$ and $\gamma$ be complex numbers with $\beta \neq 0$, and let $h(z)$ be a regular in $\bigcup$ with $h(0)=1$ and $\quad \operatorname{Re}[\beta h(z)+\gamma]>0$.

If $p(z)=1+p_{1} z+\ldots \quad$ is analytic in $\mathrm{U}$, then

$$
p(z)+\frac{z \partial_{q} p(z)}{\beta p(z)+\gamma} \prec h(z) \Rightarrow p(z) \prec h(z) .
$$

Proof: Let us assume that all functions under consideration are analytic in the closed disc $\bar{U}$. Suppose that $p(z)$ is not subordinate to $g(z)$, then by lemma 2.3 , there are $z_{0} \in \mathrm{U}, \zeta_{0} \in \partial \mathrm{U}$ and $\lambda, \lambda \geq 1$, such that

$$
p\left(z_{0}\right)+\frac{z_{0} \partial_{q} p\left(z_{0}\right)}{\beta p\left(z_{0}\right)+\gamma}=h\left(\zeta_{0}\right)+\frac{\zeta_{0} \partial_{q} h\left(\zeta_{0}\right)}{\beta h\left(\zeta_{0}\right)+\gamma} .
$$

From (2.1), we have

$$
\arg \left|\beta h\left(\zeta_{0}\right)+\gamma\right|<\frac{\pi}{2}
$$

and $\zeta_{0} \partial_{q} h\left(\zeta_{0}\right)$ is in the direction of the outer normal to the convex domain $h(\mathrm{U})$, therefore the right-hand of (2.3) is a complex number outside $h(\mathrm{U})$.

Therefore 
Hamid Shamsan and S. Latha

and this contradicts the hypothesis.

$$
p\left(z_{0}\right)+\frac{z_{0} \partial_{q} p\left(z_{0}\right)}{\beta p\left(z_{0}\right)+\gamma} \notin h(\mathrm{U})
$$

Hence $p(z) \prec h(z)$. To remove the restriction, we need to replace $p(z)$ by $p_{\tau}=p(\tau z)$ and $h_{\tau}=h(\tau z), 0<\tau<1$. All the hypotheses of the theorem are satisfied and hence we conclude that $p_{\tau}(z) \prec h_{\tau}(z)$ for each $\tau$. By letting $\tau \rightarrow 1$, we get $p(z) \prec h(z)$.

Lemma 2.5. Let $\beta$ and $\gamma$ be complex numbers with $\beta \neq 0$, and let $h(z)$ be regular in $\mathrm{U}$ with $h(0)=1$ and $\operatorname{Re}[\beta h(z)+\gamma]>0$. Let $p(z)$ is analytic in $\mathrm{U}, p(0)=1$ and satisfies

$$
p(z)+\frac{z \partial_{q} p(z)}{\beta p(z)+\gamma} \prec h(z) .
$$

and $g(z)$ is the analytic solution of

$$
g(z)+\frac{z \partial_{q} g(z)}{\beta g(z)+\gamma}=h(z),
$$

then $g(z)$ is univalent, $p(z) \prec g(z) \prec h(z)$.

Proof: Let us assume that all functions under consideration are analytic in the closed disc U. Then (2.5) and Lemma 2.4 imply $p(z) \prec h(z)$ and $g(z) \prec h(z)$. If $p(z)^{\circ} h(z)$, then by lemma 2.3 there are $z_{0} \in \mathrm{U}, \zeta_{0} \in \partial \mathrm{U}$ and $\lambda, \lambda \geq 1$, such that

From (2.4) and (2.5), we get

$$
p\left(z_{0}\right)+\frac{z_{0} \partial_{q} p\left(z_{0}\right)}{\beta p\left(z_{0}\right)+\gamma}=g\left(\zeta_{0}\right)+\frac{\lambda \zeta_{0} \partial_{q} g\left(\zeta_{0}\right)}{\beta g\left(\zeta_{0}\right)+\gamma} .
$$

$$
p\left(z_{0}\right)+\frac{z_{0} \partial_{q} p\left(z_{0}\right)}{\beta p\left(z_{0}\right)+\gamma}=g\left(\zeta_{0}\right)+\lambda\left[h\left(\zeta_{0}\right)-g\left(\zeta_{0}\right)\right] \in h(\mathrm{U}) .
$$

Now $h(\mathrm{U})$ is a convex domain, and $h\left(\zeta_{0}\right) \in \partial h(\mathrm{U}), \lambda \geq 1$ and $\left[h\left(\zeta_{0}\right)-g\left(\zeta_{0}\right)\right]$ points out of the domain. And this contradicts (2.6). Hence we conclude that $p(z) \prec g(z)$.

To remove the restriction on the functions involved, we introduce $p(\tau z), g(\tau z)$ and $h(\tau z)$. Hence the proof.

Theorem 2.6. A function $f \in \mathrm{A}$ and of the form (1.1) is in the class $k-U B_{q}(\alpha, \beta, \gamma)$ if

$$
\sum_{n=2}^{\infty} \Psi_{n}(k, \alpha, \beta, \gamma, q)<(1-\beta)(1-\gamma)
$$

where

$$
\Psi_{n}(k, \alpha, \beta, \gamma, q)=(k+1)\left\{\left([n]_{q}-1\right)(1-\alpha)(1-\gamma)+\alpha(1-\beta)[n]_{q}\left([n]_{q}-1\right)\right\}\left|a_{n}\right|
$$


On Generalized Bounded Mocanu Variation Related to $q$-Derivative and Conic Regions

$$
\begin{aligned}
& \quad+(k+1) \sum_{j=2}^{n-1}\left\{\left([j]_{q}-1\right)(1-\alpha)(1-\gamma)+[n-j]_{q} \alpha(1-\beta)\right\}[n+1-j]_{q}\left|a_{j} a_{n+1-j}\right| \\
& +(1-\beta)(1-\gamma)\left([n]_{q}+1\right)\left|a_{n}\right|+(1-\beta)(1-\gamma) \sum_{j=2}^{n-1}[n+1-j]_{q}\left|a_{j} a_{n+1-j}\right| .
\end{aligned}
$$

where $k \geq 0, \alpha \geq 0,1>\beta \geq 0,1>\gamma \geq 0,1>q>0$

Proof: Assuming that (2.7) holds, then it suffices to show that

$$
k\left|J_{q}(\alpha, \beta, \gamma, f(z))-1\right|-\operatorname{Re}\left\{J_{q}(\alpha, \beta, \gamma, f(z))-1\right\}<1 .
$$

Now, consider

$$
\begin{array}{r}
\left|J_{q}(\alpha, \beta, \gamma, f(z))-1\right|=\left|\frac{1-\alpha}{1-\beta}\left\{\frac{z \partial_{q} f(z)}{f(z)}-\beta\right\}+\frac{\alpha}{1-\gamma}\left\{1-\gamma+\frac{q z \partial_{q}^{2} f(z)}{\partial_{q} f(z)}\right\}-1\right| \\
=\left|\frac{1-\alpha}{1-\beta} \frac{z f(z) \partial_{q} f(z)}{f(z)}-\frac{(1-\alpha) \beta}{1-\beta}+\alpha+\frac{\alpha}{1-\gamma} \frac{q z \partial_{q}^{2} f(z)}{\partial_{q} f(z)}-1\right| \\
=\left|\frac{1-\alpha}{1-\beta} \frac{z f(z) \partial_{q} f(z)}{f(z)}-\frac{1-\alpha}{1-\beta}+\frac{\alpha}{1-\gamma} \frac{q z \partial_{q}^{2} f(z)}{\partial_{q} f(z)}\right| \\
=\mid \frac{(1-\alpha)(1-\gamma) z \partial_{q} f(z) \partial_{q} f(z)-(1-\alpha)(1-\gamma) f(z) \partial_{q} f(z)+\alpha(1-\beta) q z f(z) \partial_{q}^{2} f(z)}{(1-\beta)(1-\gamma) f(z) \partial_{q} f(z)} .
\end{array}
$$

Now, from (1.1) and (1.2), we have

$$
\begin{aligned}
& z \partial_{q} f(z) \partial_{q} f(z)=z\left(\sum_{n=0}^{\infty}[n]_{q} a_{n} z^{n-1}\right)\left(\sum_{n=0}^{\infty}[n]_{q} a_{n} z^{n-1}\right), a_{0}=0, a_{1}=1,[0]_{q}=[1]_{q}=1 \\
& =\frac{1}{z}\left(\sum_{n=0}^{\infty}[n]_{q} a_{n} z^{n}\right)\left(\sum_{n=0}^{\infty}[n]_{q} a_{n} z^{n}\right)=\frac{1}{z} \sum_{n=0}^{\infty}\left(\sum_{j=0}^{n}[j]_{q}[n-j]_{q} a_{j} a_{n-j}\right) z^{n} \\
& =\sum_{n=0}^{\infty}\left(\sum_{j=0}^{n}[j]_{q}[n-j]_{q} a_{j} a_{n-j}\right) z^{n-1}=z+\sum_{n=3}^{\infty}\left(\sum_{j=0}^{n}[j]_{q}[n-j]_{q} a_{j} a_{n-j}\right) z^{n-1} \\
& =z+\sum_{n=2}^{\infty}\left(\sum_{j=0}^{n+1}[j]_{q}[n+1-j]_{q} a_{j} a_{n+1-j}\right) z^{n} \\
& =z+\sum_{n=2}^{\infty}\left(2[n]_{q} a_{n}+\sum_{j=2}^{n-1}[j]_{q}[n+1-j]_{q} a_{j} a_{n+1-j}\right) z^{n} .
\end{aligned}
$$

Similarly, we can get

And

$$
f(z) \partial_{q} f(z)=z+\sum_{n=2}^{\infty}\left(\left([n]_{q}+1\right) a_{n}+\sum_{j=2}^{n-1}[n+1-j]_{q} a_{j} a_{n+1-j}\right) z^{n}
$$


Hamid Shamsan and S. Latha

$$
f(z) \partial_{q}^{2} f(z)=\sum_{n=2}^{\infty}\left([n]_{q}\left([n]_{q}-1\right) a_{n}+\sum_{j=2}^{n-1}[n+1-j]_{q}[n-j]_{q} a_{j} a_{n+1-j}\right) z^{n} .
$$

Using the equalities (2.8), we get

$$
\begin{aligned}
& \left|J_{q}(\alpha, \beta, \gamma, f(z))-1\right|=\frac{\sum_{n=2}^{\infty}\left[\left([n]_{q}-1\right)(1-\alpha)(1-\gamma)+q \alpha(1-\beta)[n]_{q}\left([n]_{q}-1\right)\right]_{n} z^{n}}{(1-\beta)(1-\gamma)\left[z+\sum_{n=2}^{\infty}\left[\left([n]_{q}+1\right) a_{n}+\sum_{j=2}^{n-1}[n+1-j]_{q} a_{j} a_{n+1-j}\right] z^{n}\right]} \\
& +\frac{\sum_{n=2}^{\infty}\left[\sum_{j=2}^{n-1}\left([j]_{q}-1\right)[n+1-j]_{q}(1-\alpha)(1-\gamma) a_{j} a_{n+1-j}\right] z^{n}}{(1-\beta)(1-\gamma)\left[z+\sum_{n=2}^{\infty}\left[\left([n]_{q}+1\right) a_{n}+\sum_{j=2}^{n-1}[n+1-j]_{q} a_{j} a_{n+1-j}\right] z^{n}\right]} \\
& +\frac{\sum_{n=2}^{\infty}\left[\sum_{j=2}^{n-1}[n+1-j]_{q}[n-j]_{q} q \alpha(1-\beta) a_{j} a_{n+1-j}\right] z^{n}}{(1-\beta)(1-\gamma)\left[z+\sum_{n=2}^{\infty}\left[\left([n]_{q}+1\right) a_{n}+\sum_{j=2}^{n-1}[n+1-j]_{q} a_{j} a_{n+1-j}\right] z^{n}\right]} \\
& \leq \frac{\sum_{n=2}^{\infty}\left|\left([n]_{q}-1\right)(1-\alpha)(1-\gamma)+q \alpha(1-\beta)[n]_{q}\left([n]_{q}-1\right)\right|\left|a_{n}\right|}{(1-\beta)(1-\gamma)\left[1-\sum_{n=2}^{\infty}\left([n]_{q}+1\right)\left|a_{n}\right|-\sum_{n=2}^{\infty}\left|\sum_{j=2}^{n-1}[n+1-j]_{q} a_{j} a_{n+1-j}\right|\right]} \\
& +\frac{\sum_{n=2}^{\infty}\left|\sum_{j=2}^{n-1}\left([j]_{q}-1\right)[n+1-j]_{q}(1-\alpha)(1-\gamma) a_{j} a_{n+1-j}\right|}{(1-\beta)(1-\gamma)\left[1-\sum_{n=2}^{n-1}\left([n]_{q}+1\right)\left|a_{n}\right|-\sum_{n=2}^{\infty}\left|\sum_{j=2}^{n-1}[n+1-j]_{q} a_{j} a_{n+1-j}\right|\right]}
\end{aligned}
$$

Since

$$
\begin{aligned}
& k\left|J_{q}(\alpha, \beta, \gamma, f(z))-1\right|-\mathfrak{R}\left\{J_{q}(\alpha, \beta, \gamma, f(z))-1\right\} \\
\leq & (k+1)\left|J_{q}(\alpha, \beta, \gamma, f(z))-1\right|
\end{aligned}
$$


On Generalized Bounded Mocanu Variation Related to $q$-Derivative and Conic Regions

$$
\begin{aligned}
& \leq \frac{(k+1) \sum_{n=2}^{\infty}\left|\left([n]_{q}-1\right)(1-\alpha)(1-\gamma)+q \alpha(1-\beta)[n]_{q}\left([n]_{q}-1\right)\right| a_{n} \mid}{(1-\beta)(1-\gamma)\left[1-\sum_{n=2}^{\infty}\left([n]_{q}+1\right)\left|a_{n}\right|-\sum_{n=2}^{\infty}\left|\sum_{j=2}^{n-1}[n+1-j]_{q} a_{j} a_{n+1-j}\right|\right]} \\
& +\frac{(k+1) \sum_{n=2}^{\infty}\left|\sum_{j=2}^{n-1}\left([j]_{q}-1\right)[n+1-j]_{q}(1-\alpha)(1-\gamma) a_{j} a_{n+1-j}\right|}{(1-\beta)(1-\gamma)\left[1-\sum_{n=2}^{\infty}\left([n]_{q}+1\right)\left|a_{n}\right|-\sum_{n=2}^{\infty}\left|\sum_{j=2}^{n-1}[n+1-j]_{q} a_{j} a_{n+1-j}\right|\right]} \\
& +\frac{(k+1) \sum_{n=2}^{\infty}\left|\sum_{j=2}^{n-1}[n+1-j]_{q}[n-j]_{q} q \alpha(1-\beta) a_{j} a_{n+1-j}\right|}{(1-\beta)(1-\gamma)\left[1-\sum_{n=2}^{\infty}\left([n]_{q}+1\right)\left|a_{n}\right|-\sum_{n=2}^{\infty}\left|\sum_{j=2}^{n-1}[n+1-j]_{q} a_{j} a_{n+1-j}\right|\right]} .
\end{aligned}
$$

The last expression is bounded by 1 if

$$
\begin{aligned}
& \sum_{n=2}^{\infty}(k+1)\left|\left([n]_{q}-1\right)(1-\alpha)(1-\gamma)+q \alpha(1-\beta)[n]_{q}\left([n]_{q}-1\right)\right|\left|a_{n}\right| \\
& +\sum_{n=2}^{\infty}\left\{\sum_{j=2}^{n-1}(k+1)\left|\left([j]_{q}-1\right)[n+1-j]_{q}(1-\alpha)(1-\gamma) a_{j} a_{n+1-j}\right|\right\} \\
& +\sum_{n=2}^{\infty}\left\{\sum_{j=2}^{n-1}(k+1)\left|[n+1-j]_{q}[n-j]_{q} q \alpha(1-\beta) a_{j} a_{n+1-j}\right|\right\} \\
& \left.+\sum_{n=2}^{\infty}(1-\beta)(1-\gamma)\left([n]_{q}+1\right)\left|a_{n}\right|+\sum_{n=2}^{\infty}(1-\beta)(1-\gamma) \sum_{j=2}^{n-1}[n+1-j]_{q} \mid\right) a_{j} a_{n+1-j} \mid \\
& <(1-\beta)(1-\gamma) .
\end{aligned}
$$

This complete the proof.

As $q \rightarrow 1$ in the above theorem we get the flowing result proved by Noor and Malik in [14], which reads.

Corollary 2.7. A function $f$ of the form (1.1) is in the class $k-U B(\alpha, \beta, \gamma)$ if

$$
\sum_{n=2}^{\infty} \Psi_{n}(k, \alpha, \beta, \gamma)<(1-\beta)(1-\gamma)
$$

where

$$
\begin{aligned}
& \Psi_{n}(k, \alpha, \beta, \gamma)=(k+1)\{(n-1)(1-\alpha)(1-\gamma)+n \alpha(1-\beta)(n-1)\}\left|a_{n}\right| \\
& +(k+1) \sum_{j=2}^{n-1}\{(j-1)(1-\alpha)(1-\gamma)+(n-j) \alpha(1-\beta)\}(n+1-j)\left|a_{j} a_{n+1-j}\right| \\
& +(1-\beta)(1-\gamma)(n+1)\left|a_{n}\right|+(1-\beta)(1-\gamma) \sum_{j=2}^{n-1}(n+1-j)\left|a_{j} a_{n+1-j}\right|
\end{aligned}
$$


Hamid Shamsan and S. Latha

where $k \geq 0, \alpha \geq 0,1>\beta \geq 0,1>\gamma \geq 0$.

As $q \rightarrow 1$ and for $\alpha=0$, we get the following result, proved by Shams and Kulkarni [15].

Corollary 2.8. A function $f \in \mathrm{A}$ and of the form (1.1) in the class $\operatorname{SD}(k, \beta)$, if it satisfies the condition

$$
\begin{aligned}
& (1-\beta)(1-\gamma)> \\
& \sum_{n=2}^{\infty}\left\{(k+1)(n-1)(1-\gamma)\left|a_{n}\right|+(k+1) \sum_{j=2}^{n-1}(j-1)(1-\gamma)(n+1-j)\left|a_{j} a_{n+1-j}\right|\right\} \\
& +\sum_{n=2}^{\infty}\left\{(1-\beta)(1-\gamma)\left|a_{n}\right|+(1-\beta)(1-\gamma) \sum_{j=2}^{n-1}(n+1-j)\left|a_{j} a_{n+1-j}\right|\right\} \\
& >(1-\gamma) \sum_{n=2}^{\infty}\{(k+1)(n-1)+(1-\beta)\}\left|a_{n}\right| .
\end{aligned}
$$

This implies that

$$
\sum_{n=2}^{\infty}\{n(k+1)-(k+\beta)\}\left|a_{n}\right|<1-\beta .
$$

As $q \rightarrow 1$ and for $\alpha=1$, we have the following result, proved by Shams and Kulkarni [15].

Corollary 2.9. A function $f \in \mathrm{A}$ and of the form (1.1) in the class $K D(k, \gamma)$, if it satisfies the condition

$$
(1-\beta)(1-\gamma)
$$

$>\sum_{n=2}^{\infty}\left\{n(k+1)(n-1)(1-\beta)\left|a_{n}\right|+(k+1) \sum_{j=2}^{n-1}(1-\beta)(n-j)(n+1-j)\left|a_{j} a_{n+1-j}\right|\right\}$

$+\sum_{n=2}^{\infty}\left\{n(1-\beta)(1-\gamma)\left|a_{n}\right|+(1-\beta)(1-\gamma) \sum_{j=2}^{n-1}(n+1-j)\left|a_{j} a_{n+1-j}\right|\right\}$

$>(1-\beta) \sum_{n=2}^{\infty} n\{(k+1)(n-1)+(1-\gamma)\}\left|a_{n}\right|$.

This implies that

$$
\sum_{n=2}^{\infty} n\{n(k+1)-(k+\gamma)\}\left|a_{n}\right|<1-\gamma .
$$

When $\beta=0, \gamma=0$ and as $q \rightarrow 1$, we get the well-known Kanas's result [10].

Corollary 2.10. A function $f \in \mathrm{A}$ and of the form (1.1) in the class $U M(\alpha, k)$, if it satisfies the condition 
On Generalized Bounded Mocanu Variation Related to $q$-Derivative and Conic Regions

$$
\begin{gathered}
\sum_{n=2}^{\infty} \Psi_{n}(k, \alpha)<1, \\
\Psi_{n}(k, \alpha)=(k+1)(n-1)(1-\alpha+n \alpha)\left|a_{n}\right| \\
+(k+1) \sum_{j=2}^{n-1}\{(j-1)(1-\alpha)+\alpha(n-j)\}\left|a_{j} a_{n+1-j}\right|+(n+1)\left|a_{n}\right|
\end{gathered}
$$

$$
+\sum_{j=2}^{n-1}(n+1-j)\left|a_{j} a_{n+1-j}\right| .
$$

For $\alpha=0, \beta=0$ and as $q \rightarrow 1$, we get the below result, proved by Kanas and Wisniowska [12].

Corollary 2.11. A function $f \in \mathrm{A}$ and of the form (1.1) in the class $k-S T$, if it satisfies the condition

$$
\sum_{n=2}^{\infty}\left\{n+(k(n-1)\}\left|a_{n}\right|<1\right.
$$

As $q \rightarrow 1, \alpha=0, k=0$, then we get result, proved by Silverman in[16].

Corollary 2.12. A function $f \in \mathrm{A}$ and of the form (1.1) in the class $S^{*}(\beta)$, if it satisfies the condition

$$
\sum_{n=2}^{\infty}(n-\beta)\left|a_{n}\right|<1-\beta
$$

Theorem 2.13. If $f(z) \in k-U B_{q}(\alpha, \beta, \gamma)$. Then $f(z) \in S D_{q}(k, \zeta)$, where

$$
\zeta=\frac{(1-\beta)(1-\gamma)}{(1-\alpha)(1-\gamma)+\alpha(1-\beta)}
$$

Proof: Let $\quad \frac{1}{1-\zeta}\left\{\frac{z \partial_{q} f(z)}{f(z)}-\zeta\right\}=p(z)$,

where $p(z)$ is analytic in $U$ and $p(0)=1$. Therefore

$$
z \partial_{q} f(z)=[(1-\zeta) p(z)+\zeta] f(z)
$$

Using $q$-logarithmic differentiation of (2.13), we get

which implies that

$$
\frac{\partial_{q}\left(z \partial_{q} f(z)\right)}{z \partial_{q} f(z)}=\frac{(1-\zeta) \partial_{q} p(z)}{(1-\zeta) p(z)+\zeta}+\frac{\partial_{q} f(z)}{f(z)},
$$

$$
1+\frac{q z \partial_{q}^{2} f(z)}{\partial_{q} f(z)}=\frac{(1-\zeta) z \partial_{q} p(z)}{[(1-\zeta) p(z)+\zeta]}+(1-\zeta) p(z)+\zeta .
$$

Using (2.13) and (2.14) in (1.4), we get 
Hamid Shamsan and S. Latha

$$
\begin{aligned}
& J_{q}(\alpha, \beta, \gamma, f(z))=\frac{1-\alpha}{1-\beta}\{(1-\zeta) p(z)+\zeta-\beta\} \\
& +\frac{\alpha}{1-\gamma}\left\{(1-\zeta) p(z)+\zeta+\frac{(1-\zeta) z \partial_{q} p(z)}{[(1-\zeta) p(z)+\zeta]}-\gamma\right\} \\
& =\frac{(1-\alpha)(1-\gamma)+\alpha(1-\beta)}{(1-\gamma)(1-\beta)}\{(1-\zeta) p(z)+\zeta\}-\frac{\beta(1-\gamma)(1-\alpha+\alpha \gamma(1-\beta))}{(1-\gamma)(1-\beta)} \\
& +\frac{\alpha}{1-\gamma} \frac{(1-\zeta) z \partial_{q} p(z)}{[(1-\zeta) p(z)+\zeta]} .
\end{aligned}
$$

Now for $\zeta=\frac{(1-\beta)(1-\gamma)}{(1-\alpha)(1-\gamma)+\alpha(1-\beta)}$, we have

$$
J_{q}(\alpha, \beta, \gamma, f(z))=p(z)+\frac{z \partial_{q} p(z)}{\frac{1-\gamma}{\alpha} p(z)+\frac{\zeta}{1-\zeta}} .
$$

Since $f(z) \in k-U B_{q}(\alpha, \beta, \gamma)$, we get

$$
p(z)+\frac{z \partial_{q} p(z)}{\frac{1-\gamma}{\alpha} p(z)+\frac{\zeta}{1-\zeta}} \prec p_{k}(z)
$$

where $p_{k}(z)$ is defined by (1.5). Since $\operatorname{Re}\left\{\frac{1-\gamma}{\delta} \frac{k}{k+1}+\frac{\zeta}{1-\zeta}\right\}>0, z \in \mathrm{U}$, applying Lemma 2.5, we get

$$
\frac{1}{1-\zeta}\left\{\frac{z \partial_{q} f(z)}{f(z)}-\zeta\right\}=p(z) \prec p_{k}(z) \text {, which implies that } f(z) \in S D_{q}(k, \zeta) \text {. }
$$

Theorem 2.14. Let $f \in \mathrm{A}$. Then $f(z) \in k-U B_{q}(\alpha, \beta, \gamma), \alpha \neq 0$, if and only if, there is a function $g(z) \in k-S T_{q}$ such that

$$
f(z)=\left[m \int_{0}^{z} t^{m-1}\left(\frac{g(t)}{t}\right)^{\frac{1-\gamma}{\alpha}} d_{q} t\right]^{\frac{1}{m}}=z+\ldots
$$

where $m=1+\frac{(1-\alpha)(1-\gamma)}{\alpha(1-\beta)}$.

Proof: From (2.15), we have

$$
\left.\left(z \partial_{q} f(z)\right)(f(z))^{m-1}=z^{\prime} m-\frac{1-\gamma}{\alpha}-1\right\}(g(z))^{\frac{1-\gamma}{\alpha}} .
$$

Using $q$-logarithmic differentiation of (2.16), we get 
On Generalized Bounded Mocanu Variation Related to $q$-Derivative and Conic Regions

$$
\frac{\partial_{q}\left(z \partial_{q} f(z)\right)}{z \partial_{q} f(z)}+(m-1) \frac{\partial_{q} f(z)}{f(z)}=\left\{m-\frac{1-\gamma}{\alpha}-1\right\} \frac{1}{z}+\left(\frac{1-\gamma}{\alpha}\right) \frac{\partial_{q} g(z)}{g(z)} \text {. }
$$

This implies that

$$
1+\frac{q z \partial_{q}^{2} f(z)}{\partial_{q} f(z)}+(m-1) \frac{z \partial_{q} f(z)}{f(z)}=\left(m-\frac{1-\gamma}{\alpha}\right)+\left(\frac{1-\gamma}{\alpha}\right) \frac{z \partial_{q} g(z)}{g(z)} .
$$

Substituting for $m=1+\frac{(1-\alpha)(1-\gamma)}{\alpha(1-\beta)}$, we get

$$
\frac{1-\alpha}{1-\beta}\left\{\frac{z \partial_{q} f(z)}{f(z)}-\beta\right\}+\frac{\alpha}{1-\gamma}\left\{1-\gamma+\frac{q z \partial_{q}^{2} f(z)}{\partial_{q} f(z)}\right\}=\frac{z \partial_{q} g(z)}{g(z)} .
$$

Now from the definition, we get the required result.

Theorem 2.15. Let $f(z) \in k-U B_{q}(\alpha, \beta, \gamma)$. Then the function

$$
g(z)=z\left(\frac{f(z)}{z}\right)^{\frac{1-\alpha}{1-\beta}}\left(\partial_{q} f(z)\right)^{\frac{\alpha}{1-\gamma}}
$$

belongs to $k-S T_{q}$ for all $z \in U$.

Proof: Using $q$-logarithmic differentiation of (2.18), we get

$$
\frac{\partial_{q} g(z)}{g(z)}=\frac{1}{z}+\frac{1-\alpha}{1-\beta} \frac{z \partial_{q} f(z)}{f(z)}-\frac{1-\alpha}{1-\beta} \frac{1}{z}-\frac{\alpha}{1-\gamma} \frac{1}{z}+\frac{\alpha}{1-\gamma} \frac{q z \partial_{q}^{2} f(z)}{\partial_{q} f(z)} .
$$

This implies that

$$
\frac{z \partial_{q} g(z)}{g(z)}=\frac{1-\alpha}{1-\beta} \frac{z \partial_{q} f(z)}{f(z)}+\frac{\alpha}{1-\gamma}\left\{1-\gamma+\frac{q z \partial_{q}^{2} f(z)}{\partial_{q} f(z)}\right\},
$$

and the result follows since $f \in k-U B_{q}(\alpha, \beta, \gamma)$.

Theorem 2.16. For $\alpha>\alpha_{1} \geq 0, \quad k-U B_{q}(\alpha, \beta, \gamma) \subset k-U B_{q}\left(\alpha_{1}, \beta, \gamma\right)$.

Proof: Let $f(z) \in k-U B_{q}(\alpha, \beta, \gamma)$. Then

$$
\begin{aligned}
& J_{q}\left(\alpha_{1}, \beta, \gamma, f(z)\right) \\
= & \frac{1-\alpha_{1}}{1-\beta}\left\{\frac{z \partial_{q} f(z)}{f(z)}-\beta\right\}+\frac{\alpha_{1}}{1-\gamma}\left\{1-\gamma+\frac{q z \partial_{q}^{2} f(z)}{\partial_{q} f(z)}\right\} \\
= & \left(1-\frac{\alpha_{1}}{\alpha}\right) J_{q}(0, \beta, \gamma, f(z))+\frac{\alpha_{1}}{\alpha} J_{q}(\alpha, \beta, \gamma, f(z)) \\
= & \left(1-\frac{\alpha_{1}}{\alpha}\right) \frac{1}{1-\beta}\left\{\frac{z \partial_{q} f(z)}{f(z)}-\beta\right\}+\frac{\alpha_{1}}{\alpha}\left[\frac{1-\alpha_{1}}{1-\beta}\left\{\frac{z \partial_{q} f(z)}{f(z)}-\beta\right\}+\frac{\alpha}{1-\gamma}\left\{1-\gamma+\frac{q z \partial_{q}^{2} f(z)}{\partial_{q} f(z)}\right\}\right]
\end{aligned}
$$


Hamid Shamsan and S. Latha

$=\left(1-\frac{\alpha_{1}}{\alpha}\right) p_{1}(z)+\frac{\alpha_{1}}{\alpha} p_{2}(z)$

$=p(z)$,

where $p_{1}(z)=\frac{1}{1-\beta}\left\{\frac{z \partial_{q} f(z)}{f(z)}-\beta\right\} \in P(q)$

and

$$
p_{2}(z)=\frac{\alpha_{1}}{\alpha}\left[\frac{1-\alpha_{1}}{1-\beta}\left\{\frac{z \partial_{q} f(z)}{f(z)}-\beta\right\}+\frac{\alpha}{1-\gamma}\left\{1-\gamma+\frac{q z \partial_{q}^{2} f(z)}{\partial_{q} f(z)}\right\}\right] \in P(q) .
$$

Now $f(z) \in k-U B_{q}(\alpha, \beta, \gamma)$, therefore

$$
J_{q}(\alpha, \beta, \gamma, f(z))=p(z) \prec p_{k}(z) .
$$

Using Theorem 2.13, we obtain

$$
J_{q}(0, \beta, \gamma, f(z)) \prec p_{k}(z) \text {. }
$$

Using these along with lemma 1.3 , we get

$$
J_{q}\left(\alpha_{1}, \beta, \gamma, f(z)\right) \prec p_{k}(z),
$$

which implies that $f(z) \in k-U B_{q}\left(\alpha_{1}, \beta, \gamma\right)$.

The $q$-Bernardi integral operator is defined as the following: Let $f(z) \in \mathrm{A}$. Then $L_{B}: \mathrm{A} \rightarrow \mathrm{A}$ is said to be $q$-Bernardi operator $L_{B}(f)=F_{a}$, and

$$
F_{a}(z)=\frac{[a+1]_{q}}{z^{a}} \int_{0}^{z} t^{a-1} f(t) d_{q} t=\sum_{n=1}^{\infty}\left(\frac{[a+1]_{q}}{[a+n]_{q}}\right) a_{n} z^{n} .
$$

Now we prove the following.

Theorem 2.17. Let $f(z) \in k-U B_{q}(\alpha, \beta, \gamma)$. Then $F_{a}(z) \in S D_{q}(k, \zeta)$, where $\zeta$ is defined by (2.12).

Proof: From (2.19), we have

which implies that

$$
z^{a} F_{a}(z)=[a+1]_{q} \int_{0}^{z} t^{a-1} f(t) d_{q} t .
$$

$$
[a+1]_{q} f(z)=[a]_{q} F_{a}(z)+z \partial_{q} F_{a}(z) .
$$

Using $q$-logarithmic differentiation of (2.20), we get

$$
\begin{aligned}
\frac{z \partial_{q} f(z)}{f(z)}= & \frac{z \partial_{q}\left(z \partial_{q} F_{a}(z)\right)+[a]_{q} z \partial_{q} F_{a}(q z)}{z \partial_{q} F_{a}(z)+[a]_{q} F_{a}(q z)} \\
& =\frac{\frac{z \partial_{q}\left(z \partial_{q} F_{a}(z)\right)}{F_{a}(q z)}+[a]_{q} \frac{z \partial_{q} F_{a}(q z)}{F_{a}(q z)}}{\frac{z \partial_{q} F_{a}(z)}{F_{a}(q z)}+[a]_{q}} .
\end{aligned}
$$


On Generalized Bounded Mocanu Variation Related to $q$-Derivative and Conic Regions Let $\frac{z \partial_{q} F_{a}(q z)}{F_{a}(q z)}=(1-\zeta) p(z)+\zeta$ with $p(z)$ is analytic in $U$ and $p(z)=1$. Then $z \partial_{q} F_{a}(z)=[(1-\zeta) p(z)+\zeta] F_{a}(q z)$

Using $q$-differentiation, we get

$$
\partial_{q}\left(z \partial_{q} F_{a}(z)\right)=[(1-\zeta) p(z)+\zeta] F_{a}(q z)+[(1-\zeta) p(z)] F_{a}\left(q^{2} z\right)
$$

which implies

$$
\frac{z \partial_{q}\left(z \partial_{q} F_{a}(z)\right)}{F_{a}(q z)}=[(1-\zeta) p(z)+\zeta]^{2}+\left[(1-\zeta) z \partial_{q} p(z)\right] \frac{F_{a}\left(q^{2} z\right)}{F_{a}(q z)} .
$$

But $\frac{F_{a}\left(q^{2} z\right)}{F_{a}(q z)}=q^{a}$ by (2.19), therefore

$$
\frac{z \partial_{q}\left(z \partial_{q} F_{a}(z)\right)}{F_{a}(q z)}=[(1-\zeta) p(z)+\zeta]^{2}+\left[(1-\zeta) q^{a} z \partial_{q} p(z)\right]
$$

Using (2.22) in (2.21), we get

$$
\begin{aligned}
\frac{z \partial_{q} f(z)}{f(z)} & =\frac{[(1-\zeta) p(z)+\zeta]^{2}+\left[(1-\zeta) q^{a} z \partial_{q} p(z)\right]+[a]_{q}[(1-\zeta) p(z)+\zeta]}{(1-\zeta) p(z)+\zeta+[a]_{q}} \\
& =(1-\zeta) p(z)+\zeta+\frac{(1-\zeta) q^{a} z \partial_{q} p(z)}{(1-\zeta) p(z)+\zeta+[a]_{q}} \\
& =(1-\zeta) h(z)+\zeta
\end{aligned}
$$

where

$h(z)=p(z)+\frac{q^{a} z \partial_{q} p(z)}{(1-\zeta) p(z)+\zeta+[a]_{q}}$.

Using $q$-logarithmic differentiation of (2.23), we get

$$
1+\frac{q z \partial_{q}^{2} f(z)}{\partial_{q} f(z)}=(1-\zeta) h(z)+\zeta+\frac{(1-\zeta) z \partial_{q} h(z)}{(1-\zeta) h(z)+\zeta} .
$$

Using (2.23) and (2.25), we get

$$
\begin{aligned}
& J_{q}(\alpha, \beta, \gamma, f(z))=\frac{1-\alpha}{1-\beta}\{(1-\zeta) h(z)+\zeta-\beta\} \\
& +\frac{\alpha}{1-\gamma}\left\{(1-\zeta) h(z)+\zeta+\frac{(1-\zeta) z \partial_{q} h(z)}{(1-\zeta) h(z)+\zeta}-\gamma\right\} \\
& \cdot \frac{(1-\alpha)(1-\gamma)+\alpha(1-\beta)}{(1-\alpha)(1-\gamma)}[(1-\zeta) h(z)+\zeta]-\frac{\beta(1-\alpha)(1-\gamma)+\alpha \gamma(1-\beta)}{(1-\alpha)(1-\gamma)} \\
& +\frac{\alpha}{1-\gamma} \frac{(1-\zeta) z \partial_{q} h(z)}{(1-\zeta) h(z)+\zeta} .
\end{aligned}
$$


Hamid Shamsan and S. Latha

Now for $\zeta=1-\frac{(1-\alpha)(1-\gamma)}{(1-\alpha)(1-\gamma)+\alpha(1-\beta)}$, we have

$$
J_{q}(\alpha, \beta, \gamma, f(z))=\frac{z \partial_{q} h(z)}{\frac{1-\gamma}{\alpha} h(z)+\frac{\zeta}{1-\zeta}} .
$$

Since $f(z) \in k-U B_{q}(\alpha, \beta, \gamma)$, we have

$$
h(z)+\frac{z \partial_{q} h(z)}{\frac{1-\gamma}{\alpha} h(z)+\frac{\zeta}{1-\zeta}} \prec p_{k}(z),
$$

where $p_{k}\left(z\right.$ is defined by (1.5). Since $\mathfrak{R}\left\{\frac{1-\gamma}{\alpha} \frac{k}{k+1}+\frac{\zeta}{1-\zeta}\right\}>0, \quad z \in \mathrm{U}$, applying Lemma 2.5 , we get

$$
p(z)+\frac{q^{a} z \partial_{q} p(z)}{(1-\zeta) p(z)+\zeta+[a]_{q}}=h(z) \prec p_{k}(z) .
$$

Now as $\mathfrak{R}\left\{(1-\zeta) \frac{k}{k+1}+\zeta+[a]_{q}\right\}>0, \quad z \in \mathrm{U}$, again applying Lemma 2.5 , we get $p(z) \prec p_{k}(z)$, which implies that $F_{a}(z) \in S D_{q}(k, \zeta)$. This completes the proof.

As $q \rightarrow 1$ in the above theorem we get the flowing result proved by Noor and Malik in [14].

Corollary 2.18. Let $f(z) \in k-U B(0, \beta, \gamma)$. Then $F_{a}(z) \in S D(k, \zeta)$, where $\zeta$ is defined by (2.12).

As $q \rightarrow 1$ and for $\alpha=0$, we get the following result, proved by Shams and Kulkarni [15].

Corollary 2.19. Let $f(z) \in k-U B(0, \beta, \gamma)$. Then $F_{a}(z) \in S D(k, \beta)$.

As $q \rightarrow 1$ and for $\alpha=\beta=0$, we get the following result, proved by Bernardi [1].

Corollary 2.20. Let $f(z) \in k-U B(0,0, \gamma)$. Then $F_{a}(z) \in k-S T$.

As $q \rightarrow 1$ and for $k=\alpha=\beta=0$, we get the following result, proved by Bernardi [1].

Corollary 2.21. Let $f(z) \in 0-U B(0, o, \gamma)$. Then $F_{a}(z) \in S^{*}$.

Acknowledgements. The authors are grateful to the reviewer and Editor-in-Chief for providing insightful comments and highly appreciate the thorough review. 
On Generalized Bounded Mocanu Variation Related to $q$-Derivative and Conic Regions

\section{REFERENCES}

1. S.D.Bernardi, Convex and starlike univalent functions, Trans. Amer. Math. Soc., 135 (1969) 429-446.

2. P.Eenigenburg, P.T.Mocanu, S.S.Miller and M.O.Reade, On a Briot-Bouquet differential subordination, In General Inequalities 3. Birkhuser Basel, (1983), 339348.

3. H.Exton, q-hypergeometric functions and applications, Ellis Horwood Series Math. Appl., Ellis Horwood, Chichester, UK. (1983).

4. U.A.Ezeafulukwe and M.Darus, A note on q-calculus, Fasciculi Math., 55(1) (2015) 53-63.

5. H.A.Ghany, q-derivative of basic hypergeometric series with respect to parameters, Int. J. Math. Anal., 3(33-36) (2009) 1617-1632.

6. A.W.Goodman, Univalent Functions, Vol I, II. Washington, New Jersey: Polygonal Publishing House, (1983).

7. I.S.Jack, Functions starlike and convex of order alpha, J. Lond. Math. Soc., 2(3) (1971) 469-474.

8. F.H.Jackson, On q-functions and a certain difference operator, Trans. Royal Soc. Edinburgh, 46 (1909) 253-281.

9. F.H.Jackson, On q-definite integrals, Quarterly J. Pure Appl. Math., 41 (1910) 193203.

10. S.Kanas, Alternative characterization of the class k-UCV and related classes of univalent functions, Serdica Math. J., 25 (1999) 341-350.

11. S.Kanas and A.Wisniowska, Conic regions and k-uniform convexity, J.Comput. Appl. Math., 105 (1999) 327-336.

12. S.Kanas and A.Wisniowska, Conic domains and starlike functions, Rev. Roumaine Math. Pures Appl., 45 (2000) 647-657.

13. P.T.Mocanu, Une propriete de conveite generlise dans la theorie de la representation conforme, Math. (Cluj), 11 (1969) 127-133.

14. K.I.Noor, and S.N.Malik, On generalized bounded Mocanu variation associated with conic domain, Math. Comput. Modelling, 55(3) (2012) 844-852.

15. S.Shams and S.R.Kulkarni and J.M.Jahangiri, Classes of uniformly starlike and convex functions, Int. J. Math. Math. Sci., 55 (2004) 2959-2961.

16. H.Silverman, Univalent functions with negative coefficients, Proc. Amer. Math. Soc., 51 (1975) 109-116.

17. A.A.Yemisci and Y.Polato $\widetilde{g}$ lu, Growth and distortion theorems for q-convex functions, Mathematica Slovaca, submitted. 